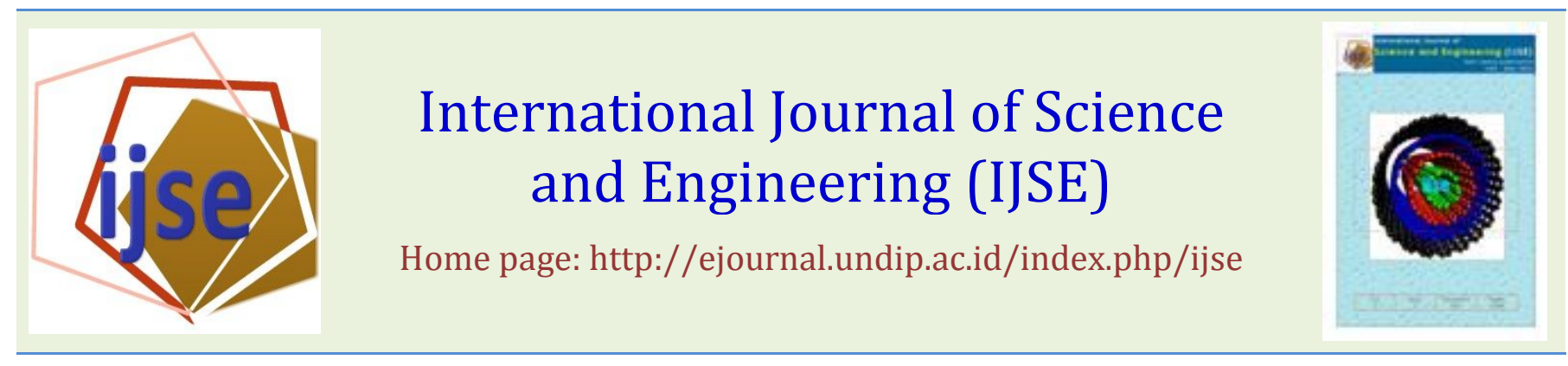

\title{
Experimental Analysis for Factors Affecting the Repeatability of Plastics Injection Molding Tests on the Self- developed Apparatus
}

\author{
Yugang Huang\#*1, Duxin Li\#2, Yuejun $\mathrm{Liu}^{* 3}$ \\ \#State Key Laboratory of Powder Metallurgy, Central South University \\ Changsha 410083, P. R. China \\ 1'hygzsh@yahoo.com, 2liduxin6404@yahoo.com \\ ${ }^{*}$ Key Laboratory of Advanced Materials and Technology for Packaging of Hunan Universities, Hunan University \\ of Technology, Zhuzhou 412007, P. R. China \\ 3yjliu_2005@126.com
}

\begin{abstract}
To improve the repeatability of the injection molding test result, the affecting factors were investigated by means of experiments. Besides the traditional processing parameter, the factors of test conditions were also considered. In order to focus on the molding process rather than the molded part, the curve measurement of the melt pressure at the entrance to the nozzle was used as the output characteristic. Experiments for polypropylene (PP) showed that the injected volume was the key processing parameter. Within the test conditions, the injection number is the most important factor. According to the analysis the operating procedure was improved effectively.
\end{abstract}

Keywords - affecting factors; plastics injection molding; repeatability; test result

Submission: May 10, 2012

Corrected : June 8, 2013

Accepted: June 10, 2013

Doi: $10.12777 /$ ijse.5.1.6-11

[How to cite this article: Huang, Y., Li, D., Liu, Y. (2013). Experimental Analysis for Factors Affecting the Repeatability of Plastics Injection Molding Tests on the Self-developed Apparatus. International Journal of Science and Engineering, 5(1),6-11. Doi: 10.12777/ijse.5.1.6-11]

\section{INTRODUCTION}

The replicated quality of the products is necessary for a plastics injection molding device, especially for an apparatus to test the injection molding process. To improve the repeatability of the injection molding process and ensure the quality of the molded parts, many researches have been undertaken by investigators. Agrawal et al. (1987) classified the controlled variables with all-phase control, phase dependent control and cycleto-cycle control. Jansen et al. (1998) investigated the effect of processing conditions on shrinkage in injection molding, and found that the holding pressure was the key parameter. Liu (2001) conducted the experiments with polypropylene on the injection molding machine and concluded that the size of the gate, the melt temperature, and the width of the rib were the principal parameters affecting the sink mark formation. Debondue et al. (2004) investigated the effects of the injection temperature and other processing parameters on the weld-line mechanical properties. Kuo and Su $(2006 ; 2007)$ studied the optimal processing parameters including mold temperature, pre- plasticity amount, injection pressure, injection speed, screw speed, packing pressure, packing time and cooling time. They applied the optimization of injection molding processing parameters to enhance the quality of liquid crystal display light-guide plates. Sha et al. (2007) examined the effects of the barrel temperature, mould temperature, injection speed and distance between the micro-features on the surface quality. Similar works have been done by Attia and Alcock (2009). Wang et al. (2008) conducted the experiments to investigate the effect of process parameters and two-way interactions on sink mark depth of injection molded parts. Rizvi and Bhatnagar (2009) studied the effect of various injection molding processing parameters on cell morphology in microcellular foamed polymeric products. Nebo et al. (2012) explored the influence of some process parameters on replication of micro-feature during micro-injection molding. Most of these investigations focus on the processing parameters of the plastics injection molding, such as barrel temperature, mould temperature, injection speed, holding pressure and packing time. In our practice 
on the injection molding apparatus, the same combinations of processing parameters could not lead to the same results. During experimental tests rather than mass production, there are some other test conditions which should be taken into account for the reproducibility of the test results.

On the other hand, the characteristics for reflecting the quality of the injection molding process varied in the studies mentioned above. Jansen et al. (1998) considered the shrinkage. Liu (2001) and Wang et al. (2008) minimized the sink marks of molded parts. Debondue et al. (2004) cared the weld quality of the molded specimens with the mechanical properties. The screw outer diameter, tensile strength and twisting strength were used as the quality characteristics by Kuo and Su (2006). Rizvi and Bhatnagar (2009) observed and analyzed the cell morphology of the microcellular foamed sample. Nebo et al. (2012) and Attia and Alcock (2009) used part mass as the output parameter to reflect the variability of the parts. All these characteristics relate to the particular parts. In order to focus on the molding process rather than the molded part, the curve measurement of the melt pressure was selected as the compared characteristic in the present work.

In order to improve the repeatability of the test result on the apparatus self-developed recently, the factors affecting the plastics injection molding process were investigated in this paper. The considered factors can be classified into two types: the traditional processing parameters and the test conditions. The latter one includes injection position, injection number and plasticization time. According to the experimental analysis, the operating procedure for injection molding tests was improved to ensure repeatability of the test result.

\section{MATERIALS AND Methods}

An injection molding apparatus was recently established based on the capillary rheometer in our laboratory. It consists of a modified capillary rheometer (as shown in Fig. 1), a mold unit and a data acquisition system (as shown in Fig. 2). The operating procedure for injection molding can be concisely expressed as follows:

$>$ First of all, the plastic sample is plasticized in the barrel with the temperature control module.

$>\quad$ Next, the mold clamping is implemented manually and easily with the slope structure of the mold boards.

$>$ Thirdly, the mold is connected with the barrel by screwing down the nozzle.

$>$ Then the sample would be filled into the mold cavity under the extrusion of the plunger rod.

$>$ During the packing phase, the plunger rod moves down slowly.

$>$ After cooling the molded part, the mold is manually opened, and one cycle for the plastics injection molding is completed. To carry out the next cycle of the experiments, we can start from the mold clamping phase.

$>$ Additional, the data acquisition would be started in every cycle from the beginning of the filling phase and stopped until the specified sampling time runs out.

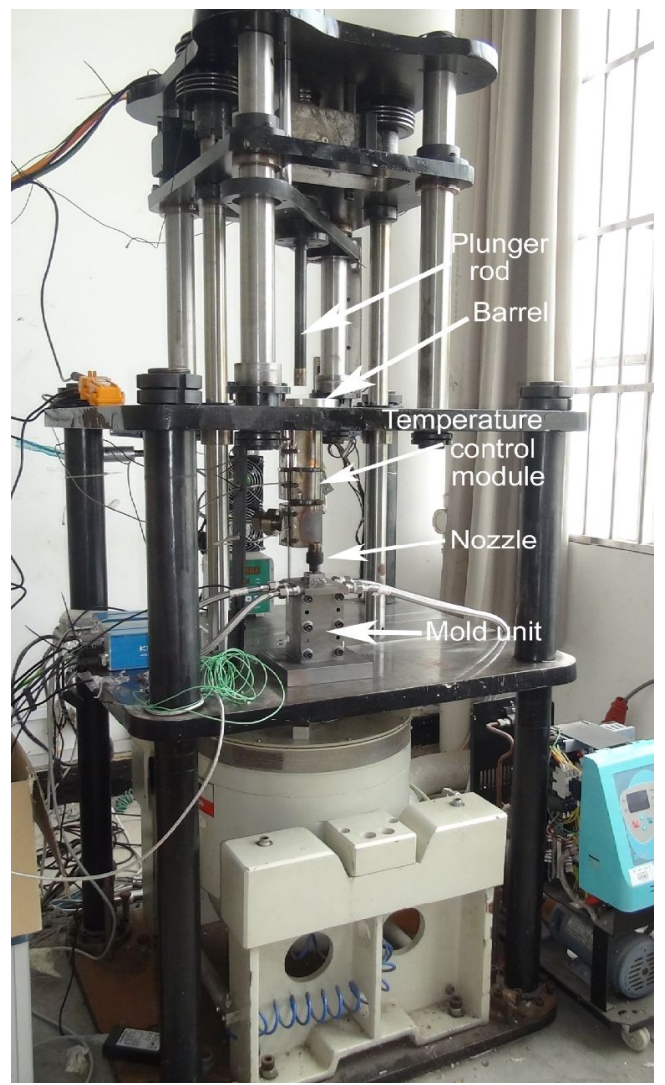

Fig. 1 Modified capillary rheometer

Fig. 2 shows the distribution of the measurement locations in the injection molding apparatus.

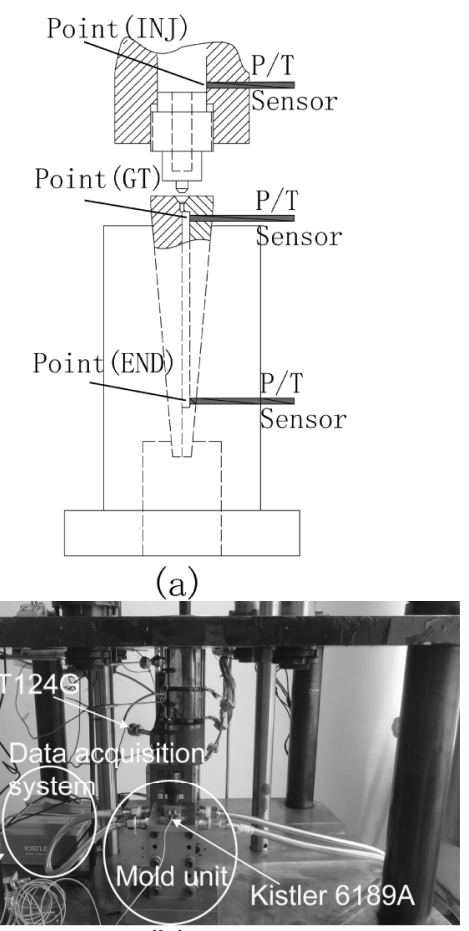

(b)

Fig. 2 Measurement locations: (a) schematic of the measurement distribution, (b) photo of the injection molding equipment 
The sample pressures and temperatures in the mold cavity are monitored by the Kistler measuring system. The system includes a Kistler CoMo Injection (Kistler Instrument AG, type 2869B, Winterthur, Switzerland) for data acquisition, two combined pressure and temperature sensors (Kistler Instrument AG, type 6189A) for the detection of cavity pressure and sample contact temperature at the same position and a melt pressure and temperature sensor (Zhaohui Pressure Apparatus Co., type PT124G-131, Shanghai, China) for the measurement of the melt pressure and temperature in the barrel. As shown in Fig. 2 (a), the injection pressure $P_{i n j}$ and the injection temperature $T_{i n j}$ are acquired near the entrance to the nozzle at the bottom of the barrel, marked with the point INJ, by the sensor PT124G-131. The pressures of $P_{g t}, P_{\text {end }}$ and the temperatures of $T_{g t}, T_{\text {end }}$ in the mold cavity are measured by the sensors Kistler $6189 \mathrm{~A}$ at the points GT and $E N D$ respectively. And all signals of these sensors are parallel acquired by the injection process monitoring system Kistler 2869B to ensure the synchronization of the original data.
All experiments were performed with a common polymer of polypropylene (PP) T30S (supplied by Sinopec Maoming Co., with MFR $3.0 \mathrm{~g} / 10 \mathrm{~min}$, at $230{ }^{\circ} \mathrm{C} / 2.16 \mathrm{~kg}$ ).

An example of the experimental result, including the molded part and the measurement data, can be seen in Fig. 3.

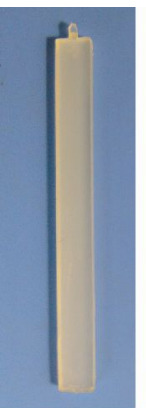

(a)

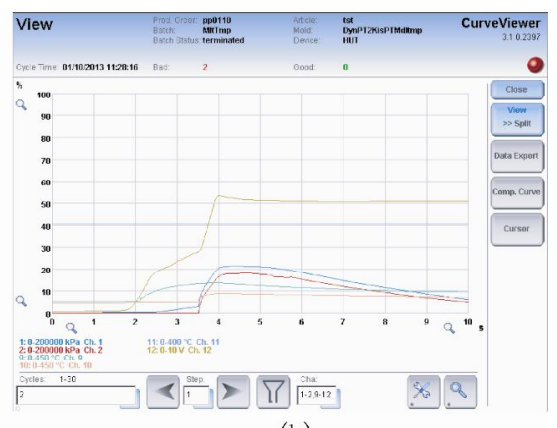

(b)
Fig. 3 Test result: (a) molded part, (b) measurement data

TABLE I

RANGE OF VALUE FOR EVERY FACTOR

\begin{tabular}{llll}
\hline Processing parameters & Range of value & Test conditions & Range of value \\
\hline Barrel temperature (i.e. & $180,185,190$, & & \\
Melt temperature) $/{ }^{\circ} \mathrm{C}$ & $200,(210), 220$ & Plasticization time $/ \mathrm{s}$ & $11,(15), 42,66$ \\
Mold temperature $/{ }^{\circ} \mathrm{C}$ & $10,(20), 40,60$, & Injection position & $130,(180), 230$ \\
Injection speed $/ \mathrm{mm} / \mathrm{s}$ & 80 & $/ \mathrm{mm}$ & $(1), 2,3,4,5$ \\
Injection distance $/ \mathrm{mm}$ & $4,6,(8), 10$ & Injection number $/-$ & \\
Packing speed $/ \mathrm{m} / \mathrm{s}$ & $16,18,20,(22)$ & & \\
Packing time $/ \mathrm{s}$ & $20,40,(50), 60$, & & \\
& 80 & & \\
& $0,3,5,(7)$ & & \\
\hline
\end{tabular}

To evaluate the effect of melt temperature on the injection molding of PP T30S, experiments were performed at melt temperatures of 155.8, 158.4, 162.3, $170.1,178.1$ and $186.2{ }^{\circ} \mathrm{C}$ (in practice, the controlled variable is the barrel temperature with the setting values of 180,185, 190, 200, 210 and $220{ }^{\circ} \mathrm{C}$ ). Meanwhile, all other factors (as shown in Table 1) remained unchanged. During the process of injection molding, melt pressures and temperatures were measured.

In the similar method, each factor was analyzed by varying its set value and keeping the other ones at the default value. All possible values of each factor are shown in Table 1, and the ones in brackets are the default. Because the driving motor of the test apparatus is the type of speed control, in this study the holding pressure of the traditional processing parameter was replaced by the moving speed of the plunger during the packing phase, namely packing speed.

\section{RESULTS AND DISCUSSION}

\section{A. Effect of processing parameters}

In order to check the effect of the melt temperature, the injection pressure $P_{i n j}$ acquired at the point INJ were measured as the functions of the time under different melt temperatures. As can be seen in Fig. 4 (a), the melt temperature mainly influences the shape of the rising segment of $P_{i n j}$ curve. To describe the difference between these rising segments, the $P_{i n j}$ values of the inflection points are plotted versus the respective melt temperature as shown in Fig. 4 (b). In test range, the maximum (45.6 $\mathrm{MPa}$ at temperature of $155.8{ }^{\circ} \mathrm{C}$ ) is almost 2.98 times of the minimum (15.3 $\mathrm{MPa}$ at temperature of $186.2{ }^{\circ} \mathrm{C}$ ), and there is the difference of $198 \%$.

Fig. 5 indicates that there were no significant differences in the injection pressure under various mold temperature. The difference between the maximum (51.6 $\mathrm{MPa}$ at mold temperature of $12.5^{\circ} \mathrm{C}$ ) and the minimum (45.5 MPa at mold temperature of $42.4^{\circ} \mathrm{C}$ ) is $13 \%$.

The effect of injection speed on the injection pressure is shown in Fig. 6. Excluding the time difference, the injection pressure mainly varies during the packing phase under different injection speeds. It ranges from 38.1 MPa to 55 $\mathrm{MPa}$, and there is the maximum difference of $44 \%$.

The injection volume is denoted by the injection distance in this study. As can be seen from Fig. 7, $P_{i n j}$ reaches $44.5 \mathrm{MPa}$ under the injection distance of $22 \mathrm{~mm}$, while it decreases to $10.9 \mathrm{MPa}$ under the injection distance of $16 \mathrm{~mm}$. There is the difference of $308 \%$. 


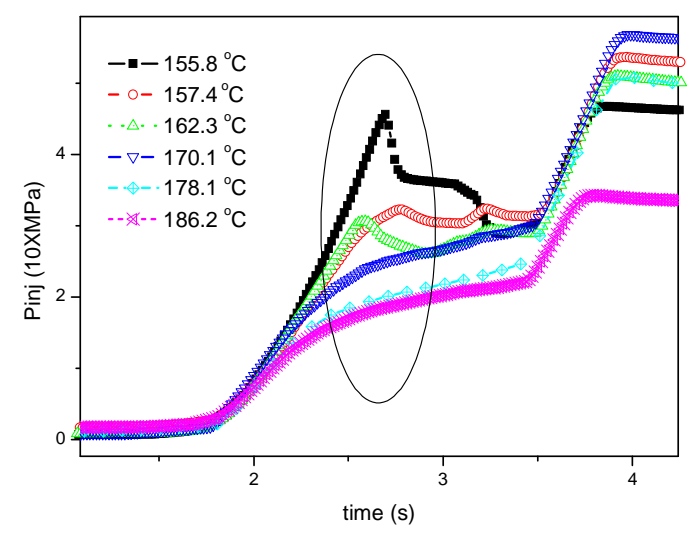

(a)

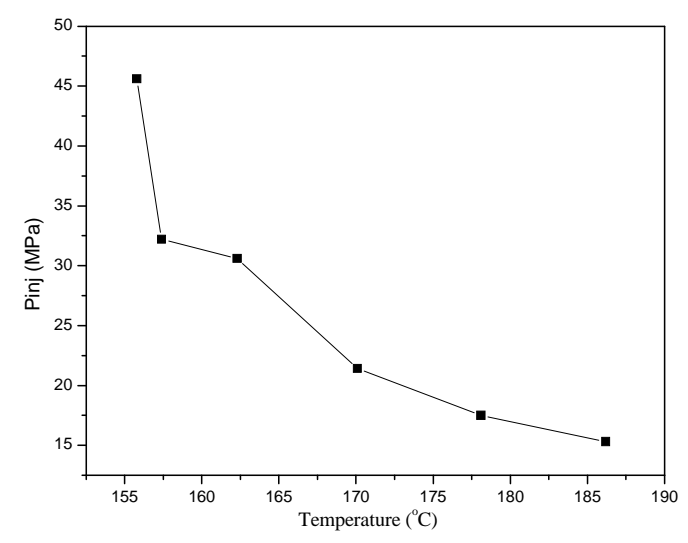

(b)

Fig. 4 Effect of melt temperature: (a) the injection pressure $P_{\text {inj }}$ functions of the time under different melt temperatures, (b) $P_{i n j}$ values at the inflection points

The effects of packing speed and packing time are shown respectively in Figures 8 and 9. Both of them mainly affect the last segment of the $P_{i n j}$ curve. The variation of packing speed within the experiments induced the difference of $33 \%$. And the packing time does not show clear effect on the $P_{i n j}$ value.

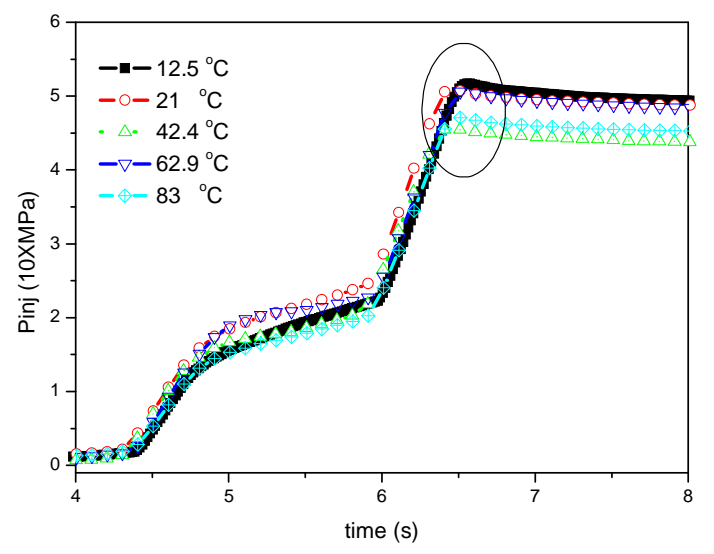

Fig. 5 Effect of mold temperature

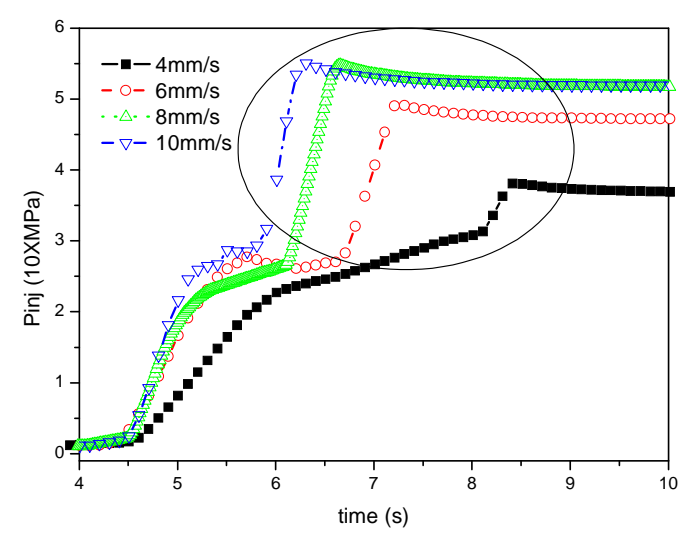

Fig. 6 Effect of injection speed

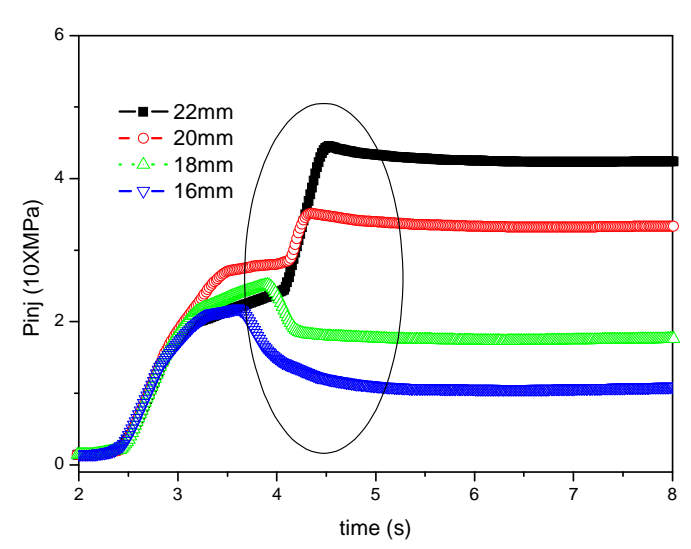

Fig. 7 Effect of injection distance

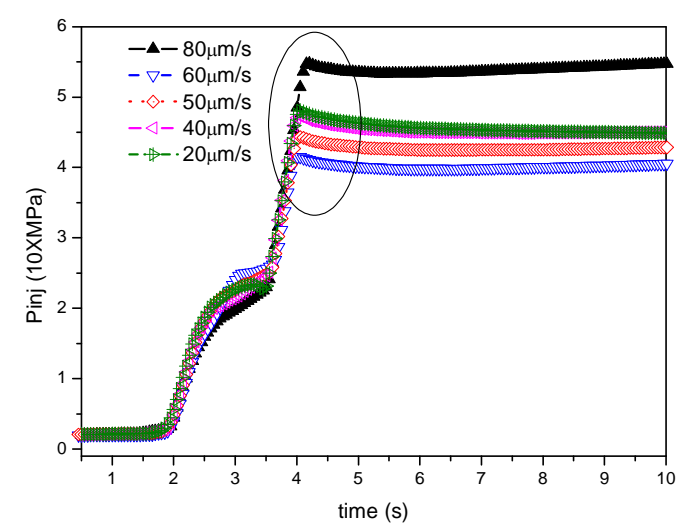

Fig. 8 Effect of packing speed

During the preliminary experiments, the test results couldn't coincide even if all processing parameters of the injection molding remain constant. Therefore, further experimental analysis was conducted to study the effect of test conditions on the repeatability of injection molding tests. 


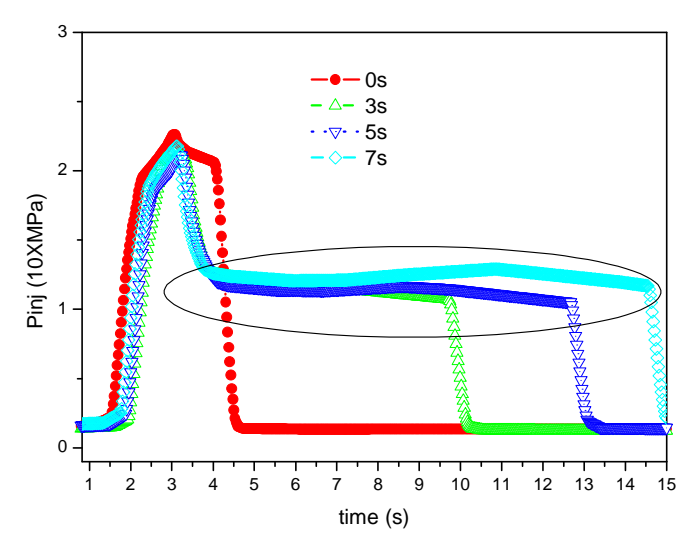

Fig. 9 Effect of packing time, under the special injection distance of 18 $\mathrm{mm}$

\section{B. Effect of test conditions}

Considering the effect of plasticization time on the rheological properties of the polymeric melt, experiments were conducted under the plasticization time of $11,15,42$ and 66 minutes. Fig. 10 shows that the plasticization time has slight effect on the injection pressure, and there is the maximum difference of $22 \%$ in the test range.

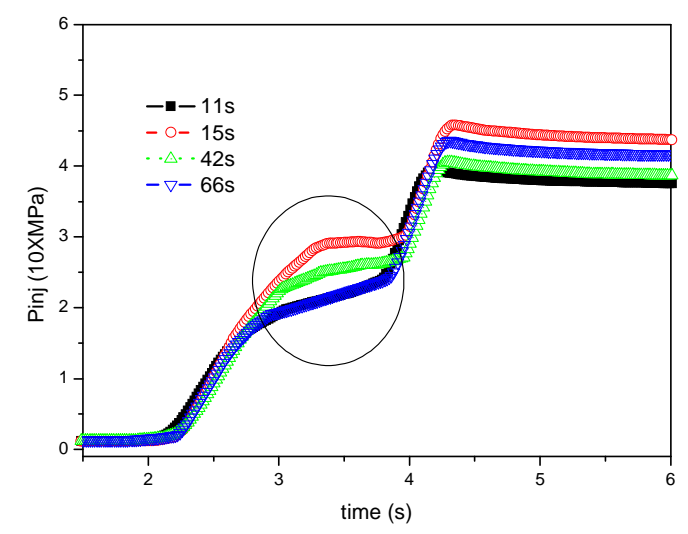

Fig. 10 Effect of plasticization time

The injection position is the displacement of the plunger with respect to the upper starting point. As the value of injection position increases, the plunger is closer to the nozzle at the bottom of the barrel, and the volume of the reservoir in the barrel is smaller. As shown in Fig.11, $P_{i n j}$ value at the inflection point increases from $31.9 \mathrm{MPa}$ to 81.8MPa when the injection position changes from 138 $\mathrm{mm}$ to $230 \mathrm{~mm}$. The difference reaches $156 \%$.

Because the volume of the reservoir in barrel is several times of the volume of the mold cavity (i.e. the sample volume), one barrel of polymeric melt could be used to perform several injection molding experiments. These experiments were numbered in accordance with their order of execution. Fig. 12 shows that the injection number is a significant factor for the injection molding process. $P_{i n j}$ value varied from 17.4 MPa to 73.2 MPa under different injections, and the maximum difference is $321 \%$.

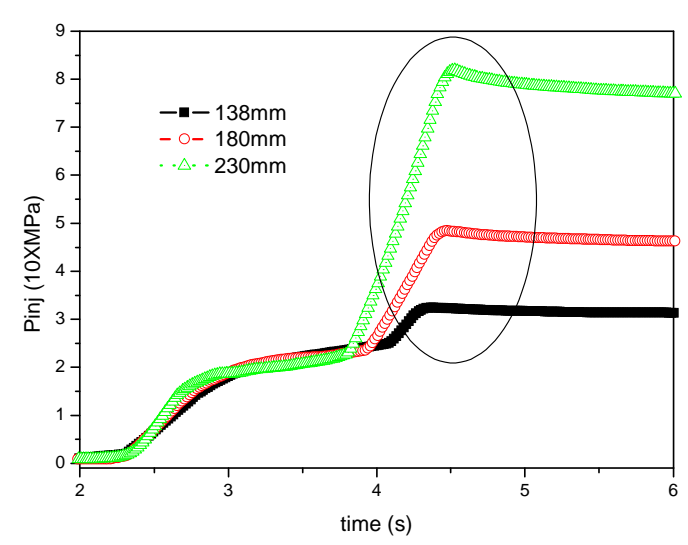

Fig. 11 Effect of injection position

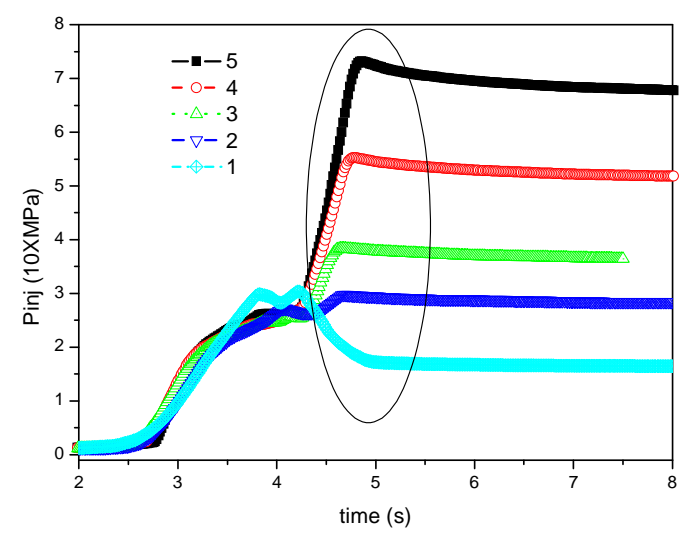

Fig. 12 Effect of injection number

\section{CONCLUSIONS}

In summary, the injection distance is the key factor of the processing parameter affecting the injection pressure $\left(P_{i n j}\right)$ curve. The effect of the melt temperature is the second most important. On the other hand, within test conditions the injection number is by far the most important factor affection the $P_{i n j}$ curve. And the injection position is slightly less important. Mold temperature, packing time and plasticization time do not show significant influence on the injection pressure in test range.

In order to ensure the repeatability of test result, the following operating precautions must be taken during the injection molding test on the self-developed apparatus:

$\checkmark$ The injection distance (i.e. injection volume) should be precisely controlled.

$\checkmark \quad$ The injection molding could be conducted only once with one barrel of sample to avoid the change in melt density caused by the injection history.

$\checkmark \quad$ The plunger should start injecting at the same point, e.g. the injection position of $180 \mathrm{~mm}$, which makes the sample in the barrel having a consistent volume and the same compressibility.

According to the experimental analysis, the coincidence of the injection molding test can be improved with these effective correcting measures. 


\section{ACKNOWLEDGMENT}

The authors gratefully acknowledge financial support by the National Natural Science Foundation of China (Grant No. 10972076), the science and technology plan project of Hunan province (Grant No. 2011TT2028), and the project of Central South University (Grant No. 134374334000010).

\section{REFERENCES}

[1] Agrawal, A. R., Pandelidis, I., and Pecht, M. 1987. Injection-Molding Process Control-A Review. POLYMER ENGINEERING AND SCIENCE, 27(18): 1345-1357. http://dx.doi.org/10.1002/pen.760271802

[2] Attia, U. M. and Alcock, J. R. 2009. An evaluation of processparameter and part-geometry effects on the quality of filling in micro-injection moulding. MICROSYSTEM TECHNOLOGIES-MICROAND NANOSYSTEMS-INFORMATION STORAGE AND PROCESSING SYSTEMS, 15(12): 1861-1872.

http://link.springer.com/content/pdf/10.1007\%2Fs00542-0090923-1

[3] Debondue, E., Fournier, J. E., Lacrampe, M. F., and Krawczak, P. 2004. Weld-line sensitivity of injected amorphous polymers. JOURNAL OF APPLIED POLYMER SCIENCE, 93(2): 644-650. http://dx.doi.org/10.1002/app.20488

[4] Jansen, K. M. B., Van Dijk, D. J., and Husselman, M. H. 1998. Effect of Processing Conditions on Shrinkage in Injection Molding. POLYMER ENGINEERING AND SCIENCE, 38(5):838-846. http://dx.doi.org/10.1002/pen.10249
[5] Kuo, C. F. J. and Su, T. L. 2006. Optimization of multiple quality characteristics for polyether ether ketone injection molding process. FIBERS AND POLYMERS, 7(4): 404-413.

http://dx.doi.org/10.1007/BF02875773

[6] Kuo, C. F. J. and Su, T. L. 2007. Optimization of injection molding processing parameters for LCD light-guide plates. JOURNAL OF MATERIALS ENGINEERING AND PERFORMANCE, 16(5): 539-548. http://dx.doi.org/10.1007/s11665-007-9088-1

[7] Liu, S. J. 2001. Effects of processing parameters on formation sinkmarks on injection moulded parts. PLASTICS, RUBBER AND COMPOSITES, 30(4): 170-174.

http://dx.doi.org/10.1179/146580101101541598

[8] Nebo, S., Ali, Z., and Scott, S. 2012. Replication of micro-feature using variety of polymer and commonly used mould at elevated temperature and pressure, INTERNATIONAL CONFERENCE ON STRUCTURAL NANO COMPOSITES (NANOSTRUC 2012), Jul. 02-04, Iop Publishing Ltd., England: 1-8.

http://iopscience.iop.org/1757-899X/40/1/012044

[9] Rizvi, S. J. A. and Bhatnagar, N. 2009. Optimization of Microcellular Injection Molding Parameters. INTERNATIONAL POLYMER PROCESSING, 24(5): 399-405.

http://dx.doi.org/10.3139/217.2263

[10] Sha, B., Dimov, S., Griffiths, C., and Packianather, M. S. 2007. Investigation of micro-injection moulding: Factors affecting the replication quality. JOURNAL OF MATERIALS PROCESSING TECHNOLOGY, 183(2-3): 284-296.

http://dx.doi.org/10.1016/j.jmatprotec.2006.10.019

[11] Wang, L. X., Li, Q., Shen, C. Y., and Lu, S. F. 2008. Effects of process parameters and two-way interactions on sink mark depth of injection molded parts by using the design of experiment method. POLYMER-PLASTICS TECHNOLOGY AND ENGINEERING, 47(1): 3035. http://dx.doi.org/10.1080/03602550701575987 